\title{
Bandwidth Optimization Through Hybrid Codecs G.711 and G.729 for VoIP Ethernet, FR and MP Networks
}

\author{
Mohamed Alahemar, Abdullah Masrub, AbdulSalam Addeeb \\ Department of Electrical \& Computer Engineering, Elmergib University, Libya
}

DOI: https://doi.org/10.21467/proceedings.2.14

* Corresponding author email: mohammed13111992@gmail.com

\begin{abstract}
With the rapid growth in both internet and telecommunication technologies, VoIP has become more and more popular and expected to replace the traditional telephony services. The main issues in communication of real time application on IP networks, however, are providing high Quality of Service (QoS), security and appropriate capacity of transmission medium. Therefore, one of the most important factors to consider when designing packet voice networks is the capacity. This paper focuses on the capacity problem and attempts to determine the minimum bandwidth (BW) that can support in each transmission rate based on different speech codecs. In precise, this paper discusses an overhead problem in VoIP transmission and studies the extent of which the required BW is affected by the type of used network and the dependency on the codec type used for the VoIP encoders. The study devises the variation payload size in two codec techniques (G.711and G.729) to optimize the BW utilization. The study concluded that increasing the payload size and using the cRTP protocol would reduce bandwidth requirements to more than $50 \%$.
\end{abstract}

Keywords: VoIP; Overhead Problem; Codec Techniques; Capacity.

\section{Introduction}

Voice over Internet Protocol (VoIP) is a technology that used widely in both internet and telecommunication fields to make voice calls and expected to replace the traditional telephony services. Nowadays, using VoIP services users are not only allowed to call other users using the same service, but they may allow to call anyone who has a subscribe number connected to a VoIP adapter. The idea of this technology is to convert the user voice into a digital signal to be transmitted using the Internet connection. The idea behind such technology is a number of protocols that organize and control connection establishment over different network layers. As the voice packet is moved over different network layers, some additional information needs to be added to the packet. Real-time Protocol (RTP), Datagram Protocol (UDP), and Internet Protocol (IP) header address represent more than $70 \%$ of the added information to such packets. The formed (RTP/UDP/IP) packet represent about 54 bytes of information. The main components of a VoIP system are CODEC (Coder-Decoder), Packetizer and playout buffer [1].

(C) 2018 Copyright held by the author(s). Published by AIJR Publisher in Proceedings of First Conference for Engineering

Sciences and Technology (CEST-2018), September 25-27, 2018, vol. 1 .
This is an open access article under Creative Commons Attribution-NonCommercial 4.0 International (CC BY-NC 4.0) license, which permits any non-commercial use, distribution, adaptation, and reproduction in any medium, as long as the original work is properly cited. ISBN: 978-81-936820-5-0 
The most critical components of a VoIP system is the voice codec. It is the process of converting the speech signal into digital form, transmit it through the medium to the receiver and reconstruct the received information to form the original signal. different algorithms are run on both sides, the sender and the receiver sides, to ensure the success deliver of the packetized voice data. Different codecs have different bit-rate, packet length, speech quality, algorithmic delay, complexity are used to enable optimization of bandwidth utilization. Bit rate is a very important parameter of codec which affects the quality of encoded speech. Therefore, to obtain the best quality of voice with the lowest bandwidth requirements, it is important to select the appropriate codec for a particular voice network [2] [3][4].

However, the main issues in communication of real time application on IP networks are providing high Quality of Service (QoS), security and appropriate capacity of transmission medium. Choosing the appropriate codec for a particular bandwidth of the network is also a little difficult. Using other internet application such as web browsing, file transfer, ... etc in the same time with VoIP service is affect the performance of the connection of the VoIP session because it is affecting the network bandwidth. Due to popularity of both the common hybrid codecs (G.711 and G.729), they have been studied in this paper to optimize the bandwidth utilization.

\section{Background}

\subsection{Voice Codecs}

With VoIP, the voice traffic is carried through the transport medium over an IP network, requiring a source, destination, User headers. Voice codec samples the waveform at regular intervals and generates a value. Samples are taken 8000 times/s (i.e. $8 \mathrm{kHz}$ sampling rate) or 16000 times/s (i.e. $16 \mathrm{kHz}$ sampling rate). The values are quantized in order to map it into discrete-finite value which can be represented using digital bits, which forms the voice data frame being transmitted over the network. To achieve such process, codec provides compression capabilities to save network bandwidth. The rapid development of VoIP technology has driven to deep advancement in designing of voice codecs which provide better QoS management capabilities. As mentioned above, choosing a proper codec is an important factor because it can affect the voice quality and bandwidth utilization together. Then the philosophy is, having higher compression codecs leads to lower bit rate which means lower bandwidth. From another point of view, high-quality voice codecs with high degree of compression require very low bandwidths for transmission, and thus have better performance in network congestion situations [2][5][6].

Formally, voice codecs are standardized by the International Telecommunication Union (ITUT). The most common voice codecs specified by ITU-T include G.711 with $64 \mathrm{kbps}$ and G.729 with $8 \mathrm{kbps}$ bit rate. Both versions are widely used. G.729 utilizes one eighth of the bandwidth compared to G.711. This means that G.729 supports more calls but they have less

Proceedings of First Conference for Engineering Sciences and Technology (CEST-2018), vol. 1 
Bandwidth Optimization Through Hybrid Codecs G.711 and G.729 for VoIP Ethernet, FR and MP Networks

quality. G.711 codec doesn't have licensing fee so it can be used in VoIP applications freely. In contrary, G.729 is a licensed codec. Most phones that support VoIP have implemented this codec in their chipset. G.711codec use Pulse Code Modulation (PCM) of voice frequencies at a standard bit rate of $64 \mathrm{Kbps}$. Typically $12 . .14$ bit samples, sampled at $8 \mathrm{KHz}$ sample rate, are compounded to 8 bit for transmission over a $64 \mathrm{Kbps}$ data channel. This codec requires low computation complexity and provides very good voice quality with negligible delay. However, it consumes $64 \mathrm{Kbps}$ of audio bandwidth per direction, which is high in comparison to other codecs. On the other hand, G.729 codec samples the voice band at $8 \mathrm{KHz}$ with a 16 bit resolution. This codec provides significant bandwidth savings. It has 8:1 compression and requires just $8 \mathrm{Kbps}$ of audio bandwidth [2][4]. Main characteristics of both codecs are shown in Table 1.

Table 1: Main characteristics of the G711, G729

\begin{tabular}{|c|c|c|l|}
\hline Codec & Data Rate (kbps) & Coding Type & \multicolumn{2}{|c|}{ Comments } \\
\hline G.711 & 64 & PCM & $\begin{array}{l}\text { Delivers precise speech transmission. } \\
\text { produces audio uncompressed }\end{array}$ \\
\hline G.729 & 8 & CS-ACELP & $\begin{array}{l}\text { Excellent bandwidth utilization. produces } \\
\text { audio compressed }\end{array}$ \\
\hline
\end{tabular}

\section{$2.2 \quad$ Related Work}

The issue of voice performance are widely studied and the most research have been done in this area focus on codec selection. The aim is to select the appropriate sampling method for suitable codec to provide better voice performance. The Quality of Service (QoS), network traffic, and bandwidth requirement are topics of interest in the research field [5]. However, not many work have been done on specific type of network and bandwidth optimization. For example, different voice codecs were employed to investigate VoIP traffic with silence suppression technique where no packets are generated in silence period [6]. Simulation methods were also used to investigate the performance of VoIP using different coding schemes [7]. As multi rate make different transmission rate and hence, different bandwidths possible. In this context, if the number of calls exceed the allocated bandwidth, the quality of perceived voice can be affected by packet loss, jitter and delay [8].

While Constant Bit Rate (CBR) traffic with different codec schemes were considered by many researchers, others suggest that G.729 codec generates smaller packets and is more error resilient than G.711 [9][10]. In such case, G.729 codec with Voice Activity Detection (VAD) enabled was used to produce the Variable Bit Rate (VBR) characteristics. Accordingly, it is more suitable for use in wireless network where there are higher channel errors [11]. Both codecs G.711 and G.729 were compared over 802.11 Distributed Coordination Function (DCF) protocol in infrastructure mode. Results have shown that the output bit rate of the G.729 encoder being eight times less than that of the G.711 encoder [12]. Moreover, to improve quality of service capabilities, it have shown that the header overhead for voice traffic 
can be reduced [13] and can also be compressed during multiplexing to increase the bandwidth efficiency [14].

\section{Bandwidth Calculation}

In order to be moved over the IP network layers, the IP packet is wrapped by the physical transmission medium. The overhead introduced in VoIP communication links by the RTP/UDP/IP headers is quite high: Consider a scenario where a G.729 codec operating at a rate of $8 \mathrm{Kbps}$, sending frames every $20 \mathrm{msec}$. This will result in a voice payloads of 20 bytes for each packet. However, to transfer these voice payloads using RTP/UDP/IP, the following headers must be added: an Ethernet header of 14 bytes (18 bytes if VLAN is used), IP header of 20 bytes, UDP header of 8 bytes and an additional 12 bytes for RTP. This is a whopping total of 54 bytes (58 with VLAN) overhead to transmit a 20-byte payload. Transmission of IP over other mediums will result in different overhead calculations. These protocol header assumptions can be summarized as follows:

- 40 bytes for: IP (20 bytes) / User Datagram Protocol (UDP) (8 bytes) / Real-Time Transport Protocol (RTP) (12 bytes) headers.

- Compressed Real-Time Protocol (cRTP) reduces the IP/UDP/RTP headers to 2 or 4 bytes (cRTP is not available over Ethernet).

- 6 bytes for Multilink Point-to-Point Protocol (MP) or Frame Relay Forum (FRF).

- 1 byte for the end-of-frame flag on MP and Frame Relay frames.

18 bytes for Ethernet L2 headers, including 4 bytes of Frame Check Sequence (FCS) or Cyclic Redundancy Check (CRC).

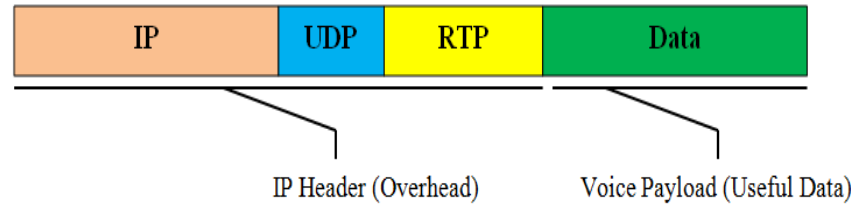

Figure 1: IP header forms a significant part of small Voice over IP packets

The amount of bandwidth required to carry voice over an IP network is dependent upon a number of factors such as Codec (coder/decoder) and sample period, IP header, Transmission medium, Silence suppression. The term 'IP header' is used to refer to the combined IP, UDP and RTP information placed in the packet, see fig. 1. The payload generated by the codec is wrapped in successive layers of information in order to deliver it to its destination. RTP is the first, or innermost, layer added. This is 12 octets. RTP allows the samples to be reconstructed in the correct order and provides a mechanism for measuring delay and jitter. UDP adds 8 octets, and routes the data to the correct destination port. It is a connectionless protocol and does not provide any sequence information or guarantee of delivery. IP adds 20 octets, and is

Proceedings of First Conference for Engineering Sciences and Technology (CEST-2018), vol. 1 
Bandwidth Optimization Through Hybrid Codecs G.711 and G.729 for VoIP Ethernet, FR and MP Networks

responsible for delivering the data to the destination host. It is connectionless and does not guarantee delivery of packets [4][5].

Overall, the IP/UDP/RTP headers add a fixed 40 octets to the payload. With a sample period of $20 \mathrm{~ms}$, the IP headers will generate an additional fixed $16 \mathrm{kbps}$ to whatever codec is being used. The payload for the G.711 codec and $20 \mathrm{~ms}$ sample period calculated above is 160 octets, the IP header adds 40 octets. This means 200 octets, or 1,600 bits are sent 50 times per second resulting in 80,000 bits per second. This is the bandwidth needed to transport the VoIP only, it does not take into account the physical transmission medium.

There are other factors, which can reduce the overhead incurred by the IP headers, such as compressed RTP (cRTP). This can be implemented on point-to-point links and reduces the IP header from 40 to just 2 or 4 octets. The codec determines the actual amount of bandwidth that the voice data will occupy. It also determines the rate at which the voice is sampled. The IP/UDP/RTP header can generally be thought of as a fixed overhead of 40 octets per packet, though on point-to-point links RTP header compression can reduce this to 2 to 4 octets (RFC 2508). The transmission medium, such as Ethernet, will add its own headers, checksums and spacers to the packet. Finally, some codecs employ silence suppression, which can reduce the required bandwidth by as much as 50 percent [2] [15].

Total packet size $=($ layer 2 header: MP or FR or Ethernet $)+($ IP/UDP/RTP header $)+$ (voice payload size)

Packet Per Second $($ PPS $)=($ codec bit rate $) /($ voice payload size $)$

Bandwidth $=$ total packet size $*$ PPS

\section{Results and Discussion}

The programming language used in this work is the Matlab, for its speed of data processing and ease of use, and the possibility of displaying the results in graphical form so that it can be easily understood. Bandwidth requirement for transmission VoIP is calculated using codecs G711, G729. We examine the effect of the used network type and the variation payload size on bandwidth requirement. In this work, we consider the types of networks (Ethernet, Frame Relay (FR), Multilink Point-to-Point Protocol (MP)), and for Payload size when using the codec $G 711$ it was $(5,10,20,30,40)$ ms while when using the codec G729 it was (10, 20, 30 , $40,50,60) \mathrm{ms}$.

From Fig. 2 and Fig. 3, it is noticed that as payload size increases the BW decreases. The network that requires the widest bandwidth is clearly the Ethernet network. This is due to not utilizing cRTP protocol which responsible for decreasing the header. On the other hand, the type of network system that requires less bandwidth is the Frame Relay (FR) and Multilink point-to-point (MP) networks. Again, this is due to cRTP function that is reduceing the header and therefore condensing the bandwidth requirements. Fig. 4 and Fig. 5 show the percentage of the header size with respect to the total load size. When increasing the voice payload size the VoIP bandwidth reduces and the overall delay increases. 


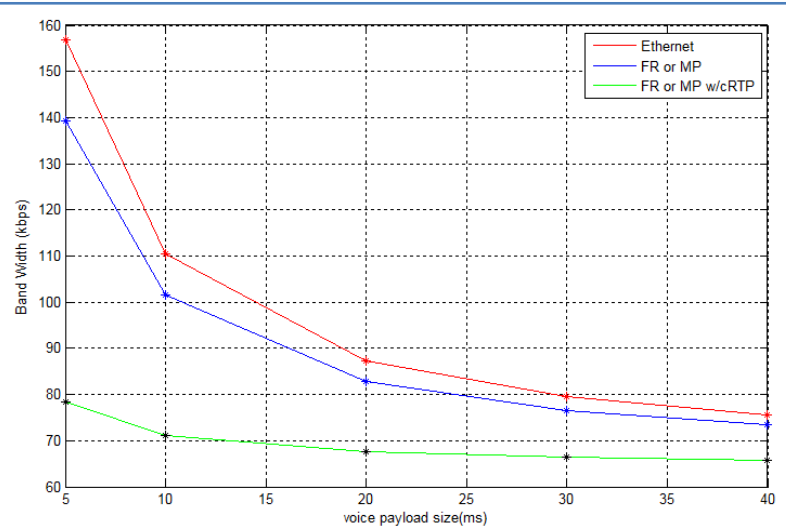

Figure 2: Bandwidth Requirement of Codec G711



Figure 3: Bandwidth Requirement of Codec G729

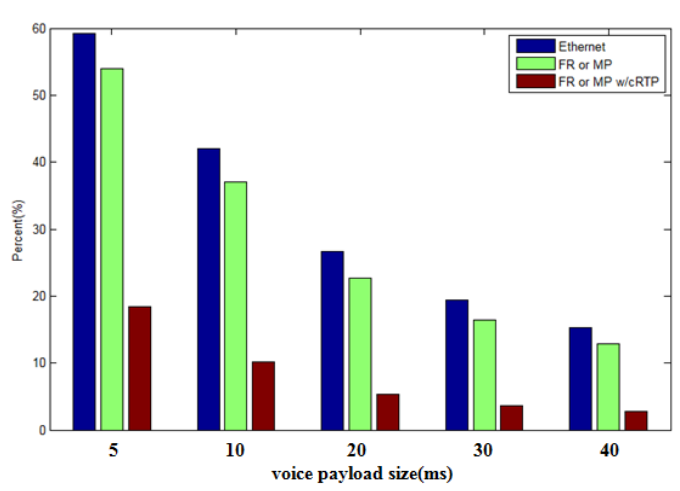

Figure 4: Packet Overhead of Codec G711

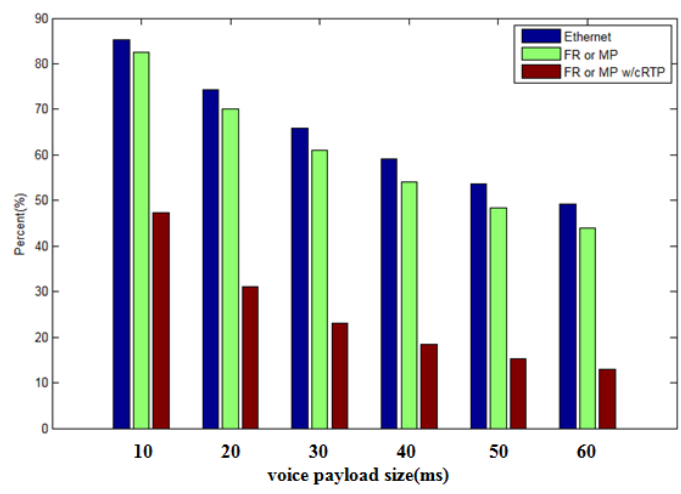

Figure 5: Packet Overhead of Codec G729

\section{Conclusions}

All VoIP packets are made up of two components: voice samples and IP/UDP/RTP headers. Although the voice samples are compressed by the Digital Signal Processor (DSP) and can vary in size based on the codec used, the headers are a constant of 40 bytes in length. With 
Bandwidth Optimization Through Hybrid Codecs G.711 and G.729 for VoIP Ethernet, FR and MP Networks

cRTP, these headers can be compressed into two or four bytes. This compression offers significant VoIP bandwidth savings. When increasing the voice payload size the VoIP bandwidth reduces and the overall delay increases.

There are many factors that influence the amount of bandwidth required to transmit a voice call over an IP network. By approaching the problem one element at a time, the final calculation becomes relatively feasible. Other factors may influence the use of the actual bandwidth, such as RTP header compression, silence suppression and other techniques are still under development. The study concluded that increasing the payload size and using the cRTP protocol would reduce bandwidth requirements to more than $50 \%$.

\section{References}

[1] H. Kazemitabar, S. Ahmed, K. Nisar, A.Said, and H. Hasbullah, "A Comprehensive review on VoIP over Wireless LAN networks, "ISSR Journal, Vol. 2, No. 2, September 2010, pp. 1-16.

[2] Haniyeh Kazemitabar and Abas Md. Said, "Capacity Analysis of G.711 and G.729 Codec for VoIP over 802.11b WLANs” International Conference, ICIEIS 2011, November 14-16, 2011, Kuala Lumpur, Malaysia.

[3] S. Rattal, A. Badri, M. Moughit, "Performance Analysis of Hybrid Codecs G.711 and G.729 over Signaling Protocols H.323 and SIP" international journal of Computer Applications, Vol. 72, No. 3, May 2013.

[4] M. Sulovic, D.Raca, M.Hadqialic and N. Hadziahmetovic "Dynamic codec selection algorithm for VoIP". The sixth international conference on digital telecommunications, ICDT 2011

[5] H.A. Ifijeh, F.E. Idachaba, and I.B. Oluwafemi, "Performance Evaluation of The Quality of VoIP Over WLAN Codecs" Proceedings of the World Congress on Engineering, July 1 - 3, 2015, London, UK.

[6] Broadcom Corporation, "Critical Steps for Successful VoIP Deployment" available at http://www.broadcom.com/ (April 2005) last visited February 2013.

[7] ] K. Salah \& A. Alkhoraidly, "An OPNET-based Simulation Approach for Deploying VoIP” International Journal of Network Management, Volume 16 Issue 3, Pages 159-183, John Wiley \& Sons, Inc. New York, NY, USA, (May 2006).

[8] M. Meeran, P. Annus, M. Alam, Y. Moullec, "Evaluation of VoIP QoS Performance in wirelessMesh Networks" Journal of information, 2017, 8, 88; doi:10.3390/info8030088.

[9] Jiango Cao \& Mark Gregory, "Performance Evaluation of VoIP Services Using Different CODECs Over A UMTS Network" Telecommunication Networks and Applications Conference, 2008. ATNAC 2008. Australasian ISBN: 978-1-4244-2602-7 pp $67-71$.

[10] Abu Sayed Chowdkery, Mark Gregory "Performance Evaluation of Heterogeneous Network for Next Generation Mobile" $200912^{\text {th }}$ International Conference on Computers and Information Technology (ICCIT 2009)Dhaka, Bangladesh, IEEE Catalog Number:CFP0917D-PRT ISBN: 978-1-4244-6281-0 pp 100-104.

[11] Bowei Xi, Hui Chen, William S. Cleveland, Thomas Telkamp, "Statistical Analysis and Modeling Of Internet VoIP Traffic for Network Engineering" Electronic Journal of Statistics Vol. 4 (2010) pp58-116 ISSN: 1935-7524 DOI: 10.1214/09-EJS473

[12] Carlos Ign’acio de Mattos, Eduardo ParenteRibeiro and Carlos Marcelo Pedroso, "A New Model For VoIP Traffic Generation" The 7th International Telecommunications Symposium (ITS 2010)

[13] AT \& T, "Critical Steps for Successful VoIP Deployment" (2005).

[14] Di Wu, "Performance Studies of VoIP over Ethernet LANs" A Master Degree dissertation submitted to Auckland University of Technology (2008).

[15] R. Dimova, G.Georgiev, Z. Stanshev "Performance Analysis of QoS Parameters for Voice over IP Applications in a LAN Segment”International Scientific Conference Computer Science'2008 